\title{
Beneficial effect of ER stress preconditioning in protection against FFA-induced adipocyte inflammation via XBP1 in 3T3-L1 adipocytes
}

\author{
Min Wang ${ }^{1} \cdot$ Xi Chen ${ }^{2} \cdot$ Zhenda Zheng $^{1} \cdot$ Shujie Yu ${ }^{1} \cdot$ Bin Zhou ${ }^{1} \cdot$ Yong Liu ${ }^{1} \cdot$ Dinghui Liu ${ }^{1} \cdot$ Yanming Chen ${ }^{3}$. \\ Xiaoxian Qian ${ }^{1,4}$
}

Received: 25 January 2019 / Accepted: 12 September 2019 / Published online: 16 October 2019

(c) The Author(s) 2019

\begin{abstract}
Adipose tissue inflammation is closely associated with the development of obesity and insulin resistance. Free fatty acids (FFAs) are a major inducer of obesity-related insulin resistance. Previously, we reported that endoplasmic reticulum (ER) stress potentially mediated retinal inflammation in diabetic retinopathy. The unfolded protein response (UPR) protects cells against damage induced by oxidative stress. X-box binding protein 1 (XBP1) plays a major role in protecting cells by modulating the UPR. However, the link between ER stress and adipocyte inflammation has been poorly investigated. In the present study, we found that pretreatment of 3T3-L1 adipocytes with a low dose of ER stress inducer tunicamycin inhibited FFAinduced upregulated expression of inflammatory cytokines. In addition, FFAs induced phosphorylation of the p65 subunit of NF- $\mathrm{KB}$ was largely inhibited by pretreatment with tunicamycin in 3T3-L1 adipocytes. Knockdown of XBP1 by siRNA markedly mitigated the protective effects of preconditioning against inflammation. Conversely, overexpression of XBP1

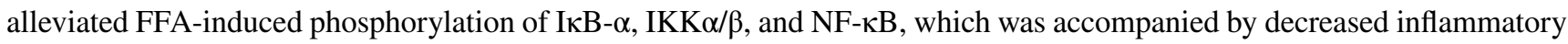
cytokine expression. Collectively, these results imply a beneficial role of ER stress preconditioning in protecting against FFAinduced 3T3-L1 adipocyte inflammation, which is likely mediated through inhibition of the IKK/NF- $\mathrm{kB}$ pathway via XBP1.
\end{abstract}

Keywords Endoplasmic reticulum stress $\cdot$ Inflammation $\cdot$ Protective effects $\cdot$ Free fatty acids $\cdot$ NF-KB

\section{Introduction}

Min Wang, Xi Chen, and Zhenda Zheng contributed equally to this work.

Yanming Chen

yanmingch@qq.com

$\triangle$ Xiaoxian Qian

yyqianxiaoxian@163.com

1 Department of Cardiology, The Third Affiliated Hospital of Sun Yat-sen University, Sun Yat-sen University, 600 Tianhe, Guangzhou 510630, Guangdong, China

2 Department of Rehabilitation Medicine, The Third Affiliated Hospital, Sun Yat-sen University, Guangzhou 510630, Guangdong, China

3 Department of Endocrinology, The Third Affiliated Hospital, Sun Yat-sen University, Guangzhou 510630, Guangdong, China

4 Institute Integrated Traditional Chinese and Western Medicine, Sun Yat-sen University, Guangzhou 510630, Guangdong, China
Obesity is a growing health problem worldwide, which is characterized by adipose cell enlargement and adipocyte inflammation [1-3]. Free fatty acids (FFAs) originating from increased lipolysis of visceral hypertrophic adipocytes lead to the inflammatory status of visceral fat through phosphorylation of several key proteins in the NF- $\mathrm{KB}$ signaling pathway [4-6]. Proinflammatory cytokines, such as MCP-1 and IL-6 secreted by inflamed adipose tissue, impair insulin signaling through different mechanisms and result in the development of cardiovascular disease [7], insulin resistance [8], and diabetes $[9,10]$.

The endoplasmic reticulum (ER) plays principle roles in proper folding and processing of nascent proteins. Perturbed ER functions cause accumulation of misfolded proteins, trigger the apoptotic cascade, and lead to cell apoptosis or death, the process of which is termed ER stress [11, 12]. Recent studies have showed that both ER stress and proinflammatory cytokines are associated with adipose tissue 
inflammation [13, 14]. Mammalian cells possess a specific signaling pathway known as the unfolded protein response (UPR) $[15,16]$. The UPR, which is activated by sublethal stimuli, provides adaptation to subsequent stress and prevents cell death [11]. It consists mainly of three ER stress response transducers: activating transcription factor-6, protein kinase RNA-like endoplasmic reticulum kinase, and inositol-requiring enzyme-1 $\alpha(\operatorname{IRE} 1 \alpha)[17,18]$. IRE1 $\alpha$ homodimerizes and auto-phosphorylates under ER stress, which contributes to increased protein chaperone content, improved ER biogenesis, and an enhanced secretory capacity via X-box binding protein 1 (XBP-1). XBP1 is the only known transcription factor downstream of IRE $1 \alpha$ that has previously been demonstrated to initiate the gene transcription, protein folding, and ER-associated protein degradation $[19,20]$.

A growing body of evidence has shown that preconditioning with ER stress protects various kinds of cell lines against inflammatory and oxidative responses [21-24]. Li et al. [25] reported that preconditioning with ER stress mitigates retinal endothelial inflammation through activation of the XBP1-mediated UPR. Moreover, Inagi et al. [21] showed that ER stress preconditioning ameliorates mesangioproliferative glomerulonephritis in a rat model. These findings suggest that ER stress may play a protective role against inflammatory response in different cell types.

In our previous study, we found that MCP-1 and IL-6 secretion as well as NF- $\mathrm{KB}$ phosphorylation are significantly increased by FFA treatment in 3T3-L1 adipocytes. In the present study, we aimed to elucidate the effects of ER stress preconditioning on FFA-induced inflammatory responses in 3T3-L1 adipocytes and to explore the involved molecular mechanisms. Our data showed that ER stress preconditioning suppressed FFA-induced proinflammatory cytokine secretion and attenuated FFA-induced activation of the NF-kB pathway via XBP1, a principal coordinator of the adaptive UPR. We also elucidated that XBP1 negatively regulated IRE1 phosphorylation and blocked FFA-induced activation of the IRE1/IKK/NF- $\mathrm{BB}$ pathway.

\section{Methods}

\section{Cell culture and adipogenic differentiation of 3T3-L1 preadipocytes}

Embryonic mouse fibroblast line 3T3-L1 preadipocytes were purchased from the American Type Culture Collection (Manassas, VA, USA) and cultured in high glucose Dulbecco's modified Eagle's medium (DMEM) (Invitrogen, Carlsbad, CA), supplemented with $10 \%$ bovine calf serum (Hyclone, Logan, UT) at $37{ }^{\circ} \mathrm{C}$ in a atmosphere containing $5 \% \mathrm{CO} 2$ until differentiation was induced as described previously [26]. Briefly, at 2 days post-confluency, cells were induced by differentiation medium containing $0.5 \mathrm{mM}$ isobutylmethylxanthine, $1 \mathrm{M}$ dexamethasone, $10 \mu \mathrm{g} / \mathrm{ml}$ insulin (MDI; Sigma, St. Louis, MO) for 3 days. Then, the cells were transferred to DMEM with $10 \mu \mathrm{g} / \mathrm{ml}$ insulin and the medium was changed every 2 days. Oil red staining is performed to confirm the maturation of adipocytes.

\section{Co-culture of adipocytes and macrophages}

3T3-L1 adipocytes and RAW264.7 cells were co-cultured using the transwell system as reported previously [27]. Briefly, the 3T3-L1 preadipocytes were cultured and differentiated into 3T3-L1 adipocytes in the lower chamber of the 24-well transwell plate inserts with a $0.4-\mu \mathrm{m}$ porous membrane (Corning, New York, NY). Then the 3T3-L1 adipocytes with indicated treatments were washed and replaced with DMEM contained 2\% FBS. At the same time, the RAW264.7 cells $\left(5.0 \times 10^{5}\right.$ cells/well) were cultured in the upper chamber, and then the two sets were put together by assembling two part of Transwell and incubated in DMEM with $2 \%$ FBS at $37^{\circ} \mathrm{C}$ for $4 \mathrm{~h}$.

\section{Cell treatments}

Differentiated adipocytes were serum starved for $16 \mathrm{~h}$ in DMEM supplemented with $2 \%$ FBS before treatment. Tunicamycin was obtained from Sigma-Aldrich. ER stress preconditioning was promoted as described previously [28, 29]. Briefly, the differentiated mature 3T3-L1 adipocytes were pretreated with a very low dose $(0.5 \mu \mathrm{g} / \mathrm{ml})$ of tunicamycin for $4 \mathrm{~h}$ followed by treatment with FFA for $4 \mathrm{~h}$. For the macrophage migration assay, the 3T3-L1 adipocytes were preincubated with control siRNA or XBP1siRNA transfection followed by FFA treatment for $4 \mathrm{~h}$ in the presence or absence of TM preconditioning, respectively, and then the co-culture systems were incubated in DMEM with $2 \%$ FBS at $37^{\circ} \mathrm{C}$ for $4 \mathrm{~h}$ before testing the macrophage migration.

\section{Macrophage migration assay}

The cell migration assay was performed as previously reported [30]. Briefly, non-migrated RAW264.7 cells in the upper chamber were carefully removed and migrated cells on the lower membrane surface were fixed in $1 \%$ paraformaldehyde, stained with hematoxylin, and counted (Ten random fields per well). Migrated cell counts were expressed as the mean number of cells per field of view.

\section{Preparation of fatty acid-albumin complexes}

Saturated palmitic acid was used in this study as described previously [6, 31]. Briefly, FFAs were dissolved in ethanol 
at $200 \mathrm{mmol} / \mathrm{l}$ and then combined with $10 \%$ FFA-free low endotoxin BSA at concentrations of $1-10 \mathrm{mmol} / \mathrm{l}$. Stock solutions were filter sterilized and stored at $-20{ }^{\circ} \mathrm{C}$. A control solution containing ethanol and BSA were applied as control solution. The final BSA concentration was consistently $1 \%$ in all FFA media.

\section{MCP-1 and IL-6 secretion measurements of by ELISA}

The cell culture supernatant was collected after treatments. MCP-1 and IL-6 in supernatants were quantified in triplicate by mouse ELISA kits (R\&D Systems, Wiesbaden-Nordenstadt, Germany), according to the manufacturer's instructions. Briefly, $50 \mu \mathrm{l}$ Assay Diluent was added, and then standards or sample were added to the pre-coated microtiter plate followed by incubation for $2 \mathrm{~h}$. Then, $100 \mu \mathrm{l} \mathrm{MCP}-1 /$ IL-6 conjugate was immediately added. Then, $100 \mu \mathrm{l} \mathrm{Sub-}$ strate Solution was added followed by adding $100 \mu 1$ Stop Solution. MCP-1 and IL-6 concentrations were calculated and normalized by total cell numbers.

\section{Adenovirus infection of 3T3-L1 adipocytes}

Recombinant adenoviruses expressing XBP1 (Ad-XBP1) and GFP (Ad-GFP) were purchased from Obio Technology (Shanghai, China). For adenoviral infection, 3T3-L1 cells were infected with the adenovirus encoding XBP-1 at a multiplicity of infection of 20 for $48 \mathrm{~h}$. Cells were then starved in medium containing $3 \% \mathrm{FBS}$ for $16 \mathrm{~h}$ and then subjected to various treatments. Infection with the adenovirus encoding GFP was employed as a control.

\section{RNA interference and cell transfection}

XBP-1 and control siRNAs were purchased from Qiagen (Valencia, CA). A $20 \mu \mathrm{M}$ stock solution of XBP-1 siRNA or control scramble siRNA was prepared in siRNA dilution buffer. 3T3-L1 adipocytes were transfected in 6-well plates according to the manufacturer's protocol. Briefly, for each transfection, high glucose DMEM containing 2\% FBS without antibiotics was used as the transfection medium. Transfection medium $(200 \mu \mathrm{l})$ containing $4 \mu \mathrm{l}$ siRNA stock solution was incubated with $2 \mu \mathrm{l}$ transfection reagent (Lipofectamine 2000, Invitrogen) for $45 \mathrm{~min}$ at room temperature. The siRNA-lipid complex which contained $1 \mathrm{ml}$ transfection media was then added to each well. After $6 \mathrm{~h}$ of incubation, the transfection medium was replaced with growth medium containing 4\% FBS without antibiotics for $16 \mathrm{~h}$, which was then changed to normal growth medium for an additional $24 \mathrm{~h}$ before experiments. The protein level of XBP1 determined by western blot analysis is applied to assess the knockdown efficiency.

\section{Western blot analysis}

Cells were lysed in ice-cold lysis buffer with a protease inhibitor cocktail (Santa Cruz Biotechnology, Santa Cruz, CA) for $30 \mathrm{~min}$ and then centrifuged at $13,300 \mathrm{rpm}$ for $20 \mathrm{~min}$. Thirty micrograms of protein were separated on an SDS-PAGE gel and then transferred. After blocking, the membrane was incubated overnight at $4{ }^{\circ} \mathrm{C}$ with a primary antibody including anti-phospho-NF- $\kappa \mathrm{B}$ p65 (Ser536),

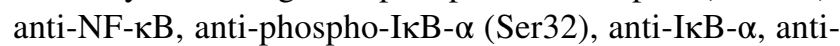
phospho-IKK $\alpha / \beta$ (Ser176/180) (Cell Signaling Technology, Boston, MA), anti-phospho-IRE1 $\alpha$ (Abcam, Cambridge, MA), anti-IRE1 $\alpha$ (Abcam, Cambridge, MA), or anti- $\beta$-actin (Cell Signaling Technology) antibodies. After incubation with an HRP-conjugated secondary antibody, signals were detected with a chemiluminescence western blotting detection solution using Bio Imaging System (Syngene, Frederick, MD).

\section{Statistical analysis}

For quantification of the western blotting results, the intensities of bands were measured using image analysis software Multi Gauge, V3.0 (FUJIFILM, Tokyo, Japan). All experiments were performed with triplicates and repeated at least three times. Statistical analyses were performed using the Student's $t$ test, ANOVA, and Bonferroni's multiple comparison test. $P<0.05$ indicated significance.

\section{Results}

\section{ER stress preconditioning attenuates the FFA-induced inflammatory response}

Induction of inflammatory cytokine expression in 3T3-L1 adipocytes is a central step in the pathogenesis of adipocyte inflammation. We first determined whether ER stress preconditioning affected FFA-induced inflammatory cytokines expression in 3T3-L1 adipocytes. ER stress preconditioning was promoted by pretreatment of differentiated mature 3T3$\mathrm{L} 1$ adipocytes with a very low dose of tunicamycin $(0.5 \mu \mathrm{g} /$ $\mathrm{ml})$ for $4 \mathrm{~h}$. Cells were then treated by FFAs $(0.5 \mathrm{mM})$ for $4 \mathrm{~h}$, followed by measurement of inflammatory protein levels. The results showed that MCP- 1 and IL-6 secretion were upregulated by 10 - and 8.5 -fold, respectively, which were markedly inhibited by tunicamycin pretreatment (Fig. 1a and $b$ ).

$\mathrm{NF}-\mathrm{KB}$ activation is a pivotal step in the overproduction of a variety of proinflammatory cytokines such as MCP-1 and IL-6. To testify whether preconditioning by ER stress affects activation of NF- $\mathrm{KB}$ in 3T3-L1 adipocytes, we analyzed phosphorylation of the p65 subunit of NF- $\kappa B$ at 
Fig. 1 Inhibition of FFAinduced proinflammatory cytokines expression by ER stress preconditioning in 3T3$\mathrm{L} 1$ adipocyte. a, b Expressions of MCP-1(a) and IL-6 (b) were determined by ELISA and normalized by cell number. 3T3-L1 adipocytes were preincubated with $0.5 \mu \mathrm{g} / \mathrm{ml}$ tunicamycin for $4 \mathrm{~h}$ followed by exposure to $0.5 \mathrm{mM}$ FFA for $4 \mathrm{~h}$. $\mathbf{c}$ Phosphorylation of NF-kB p65 subunit at Ser536 was determined by Western blot analysis and semi-quantified by densitometry. * for $P<0.05$;

** for $P<0.01$ versus control;

${ }^{\dagger}$ for $P<0.05$, , for $P<0.01$ versus FFA. Results represent three independent experiments. Values were expressed as mean $\pm \mathrm{SD}$
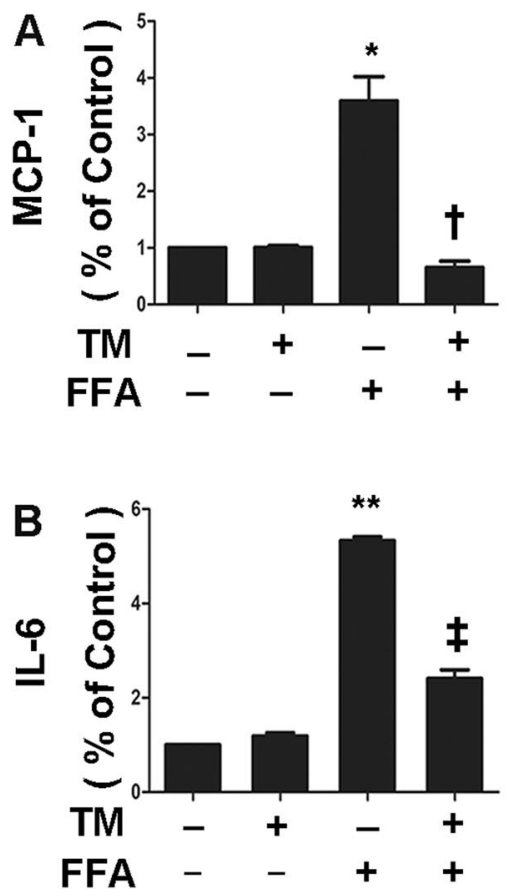

C
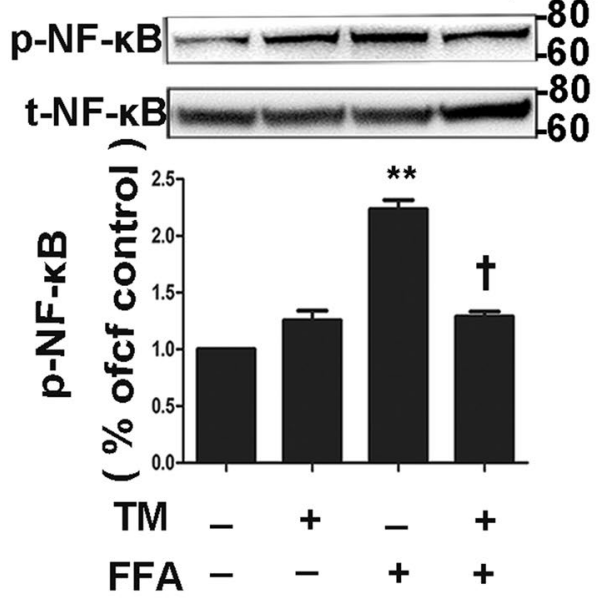

Ser536. Exposure of 3T3-L1 adipocytes to FFAs resulted in increased phosphorylation of the p65 subunit of NF- $\kappa B$ at Ser536 and the effect was largely inhibited by pretreatment with tunicamycin (Fig. 1c). This result indicates that ER stress preconditioning reduces the FFA-induced inflammatory response and NF- $\mathrm{\kappa B}$ activation in 3T3-L1 adipocytes.

\section{ER stress preconditioning upregulates XBP1 expression in 3T3-L1 adipocytes}

XBP1 activated by IRE1 is a key regulator of the adaptive UPR responding to ER stress. In addition, recent studies demonstrated that XBP1 is also an important player that mediated lipid metabolism [32,33]. Then, we determined whether XBP1 is stimulated by ER stress preconditioning inducer tunicamycin. In our study, we found the expression of XBP1 is induced by tunicamycin at a dose-dependent manner (Fig. 2a). In addition, the protein level of XBP1 is
A
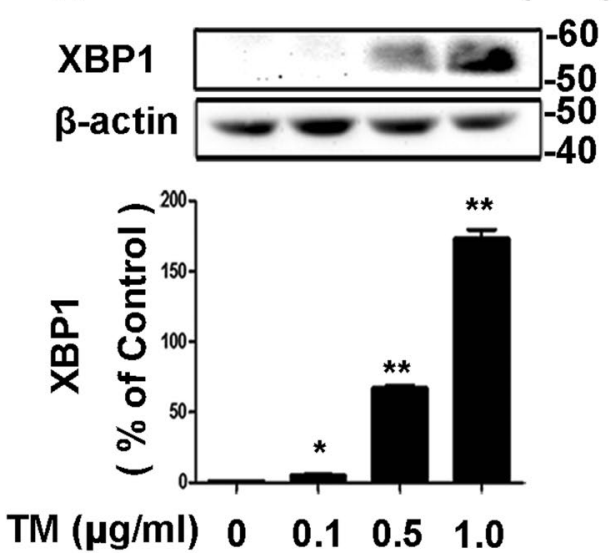

Fig. 2 Tunicamycin treatment alone upregulates the expression levels of X-box-binding protein-1 (XBP1) in 3T3-L1 adipocytes in a dosedependent and time-dependent manner. a-b The 3T3-L1 adipocytes were treated with tunicamycin $(0.5 \mu \mathrm{g} / \mathrm{ml})$ for the indicated dose (a)
B
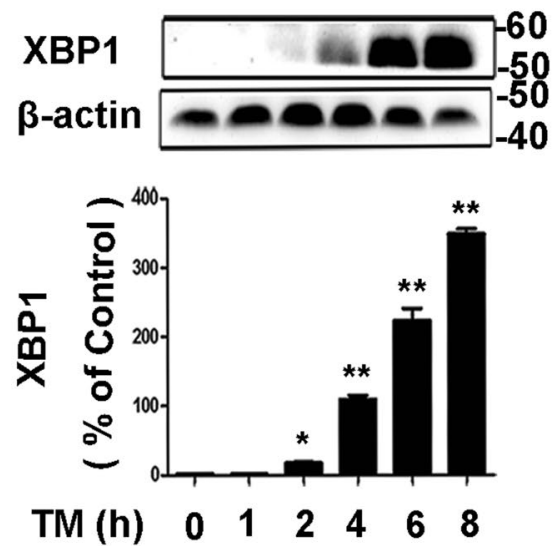

and the indicated durations (b), and a gene expression analysis of XBP1 was conducted. The results are expressed as the fold change. Results represent three independent experiments. Values were expressed as mean \pm SD. * for $P<0.05$; ** for $P<0.01$ versus control 
upregulated from $4 \mathrm{~h}$, peaked at $8 \mathrm{~h}$, and declined at $12 \mathrm{~h}$ (Fig. 2b). These results indicate that expression of XBP1 is upregulated by ER stress preconditioning inducer tunicamycin in 3T3-L1 adipocytes.

\section{Silencing XBP1 blocks the protective effects of ER stress preconditioning on inflammation and NF-KB phosphorylation}

We next determined the role of XBP1 in the anti-inflammatory effects stimulated by FFA in 3T3-L1 adipocytes. As shown in Fig. 3, pretreatment with XBP1siRNA significantly restored the secretion of MCP-1 $(P<0.05$; Fig. 3a) and IL-6 $(P<0.05$; Fig. 3b) in FFA-treated 3T3-L1 adipocytes with ER stress preconditioning. Moreover, XBP1siRNA blocked the protective effect of ER stress preconditioning on RAW264.7 macrophages migration (Fig. 4a, b) and NF- $\mathrm{kB}$ phosphorylation (Fig. 3c). These results collectively suggest that XBP1 plays a beneficial role in ER stress preconditioning in inhibiting the inflammatory response of 3T3-L1 adipocytes.

\section{XBP1 ameliorates FFA-induced inflammation and suppresses NF-KB phosphorylation}

To further verify whether XBP1 directly regulated the inflammatory response of FFA-treated 3T3-L1 adipocytes, we overexpressed XBP1 in 3T3-L1 adipocytes by infection of cells with adenovirus encoding mouse XBP1 (Ad$\mathrm{XBP} 1$ ). Adenovirus encoding GFP (Ad-GFP) was used as control. The overexpression of XBP1 in the 3T3-L1 adipocytes was confirmed by western blot analysis (data not shown). Inflammatory molecule secretion was determined by ELISAs and NF- $\kappa B$ phosphorylation was determined by western blot analysis. The results showed that FFA-induced robust increases of MCP-1 and IL-6 secretion (Fig. 5a, b), and NF- $\mathrm{KB}$ phosphorylation in Ad-GFP-treated cells, but not in Ad-XBP1-treated cells (Fig. 5c).

\section{XBP1 inhibits IKK activation and suppresses IRE phosphorylation}

IKK activation is an essential step in inflammatory pathways that lead to NF- $\mathrm{KB}$ activation. IKK upregulates the phosphorylation of the protein I $\kappa \mathrm{B}$ that lead to the nuclear translocation of NF- $\kappa B[39,40]$ and activates inflammatory response. To investigate whether NF- $\mathrm{KB}$ activation is regulated by XBP1 through IKK, we determined the effect of XBP1 on
Fig. 3 Genetic inhibition of $\mathrm{XBP} 1$ attenuated the protective effect of ER stress preconditioning in 3T3-L1 adipocyte. a-b Expressions of MCP-1 (a) and IL-6 (b) were determined by ELISA and normalized by cell number. $\mathbf{c}$ Phosphorylation of NF-кB p65 subunit at Ser536 was determined by Western blot analysis. 3T3-L1 adipocytes were transfected with XBP1siRNA for $48 \mathrm{~h}$ followed by exposure to $0.5 \mathrm{mM}$ FFA for 4 h. * for $P<0.05$ versus Ctrl. $* * P<0.01$ versus control; ${ }^{\ddagger} P<0.01$ versus FFA; ${ }^{\#} P<0.05$ versus FFA + tunicamycin
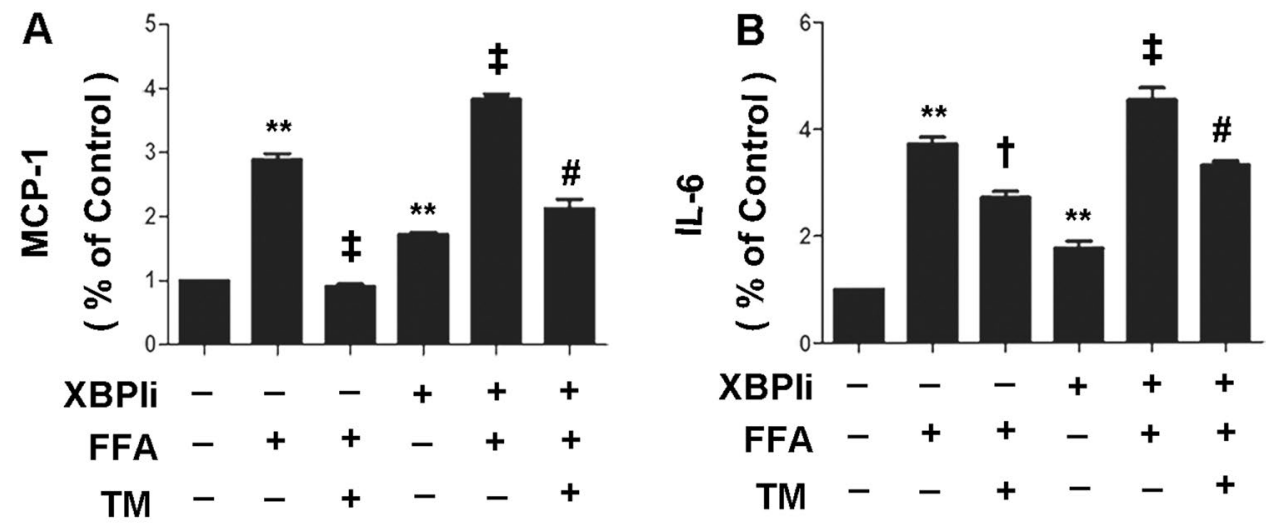

C

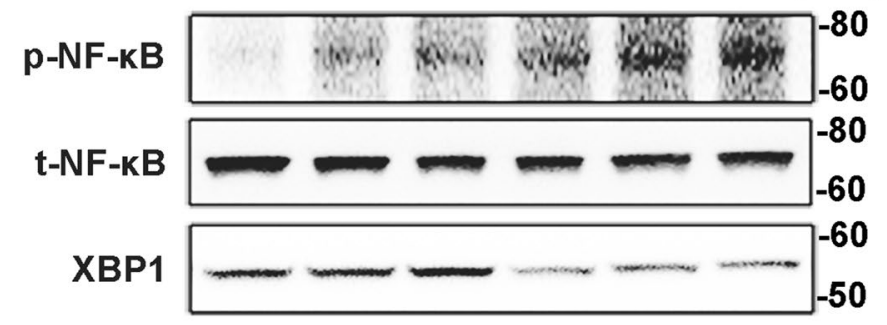



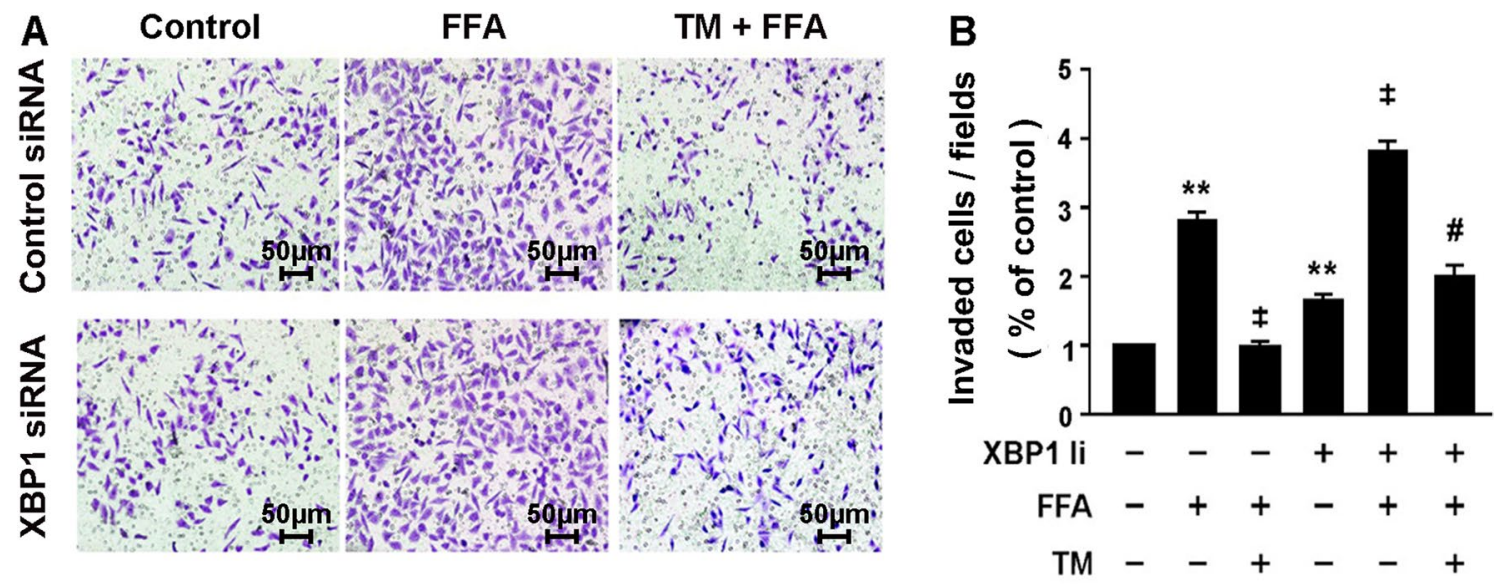

Fig. 4 Genetic inhibition of XBP1 suppressed the protective effect of ER stress preconditioning on macrophage motility. a Control siRNA (A); Control siRNA +FFA (B); Control siRNA + TM+FFA (C); D: XBP1siRNA (D); E: XBP1siRNA+FFA (E); XBP1siRNA + TM+FFA (F). Scale bar, $50 \mu \mathrm{m}$. b Quantification of migrated cells was counted as the mean number of cells per field of view. RAW 264.7 macrophages were treated with DMEM contained
2\% FBS or 3T3-L1 conditioned medium for $4 \mathrm{~h}$. Migrated RAW264.7 macrophages were quantified and expressed as the mean number of cells per field of view. A: Migrated RAW264.7 macrophages were stained with hematoxylin. Representative results were from three independent experiments. ${ }^{*}$ for $P<0.05$ versus Ctrl. $* * P<0.01$ versus control; ${ }^{\ddagger} P<0.01$ versus FFA; ${ }^{\#} P<0.05$ versus FFA + tunicamy$\operatorname{cin}$

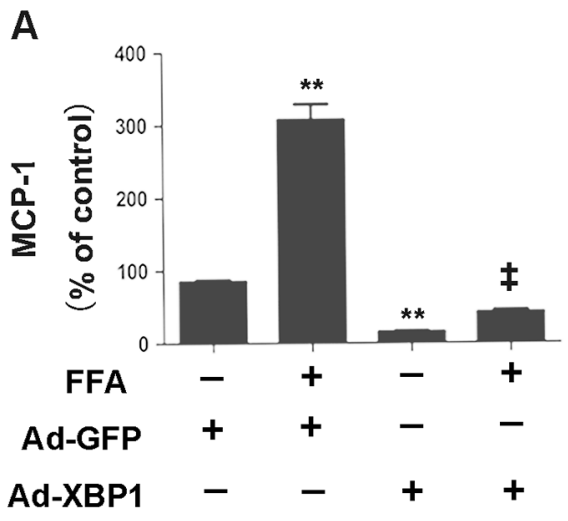

B

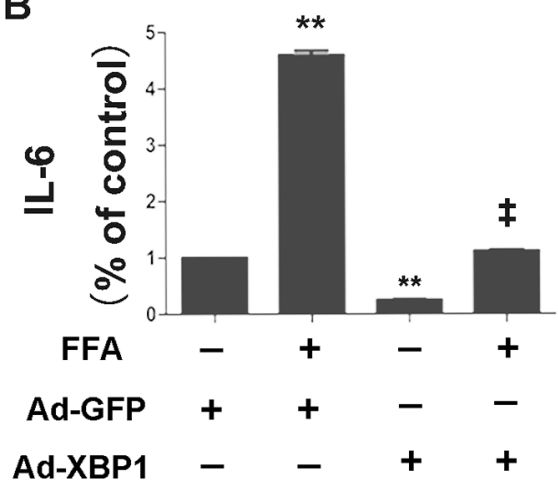

Fig. 5 Overexpression of XBP1 inhibited FFA-induced proinflammatory cytokines expression via suppression of NF- $\mathrm{BB}$ activation in 3T3-L1 adipocytes. a, b Expressions of MCP-1 (a) and IL-6 (b) were determined by ELISA and normalized by cell number. c Phosphorylation NF- $\kappa$ B p65 (Ser536) was determined in 3T3-L1 adipocytes after treatment with FFA for $4 \mathrm{~h}$. Total NF-kB was also determined by Western blot analysis and semi-quantified by densitometry. Over-
C
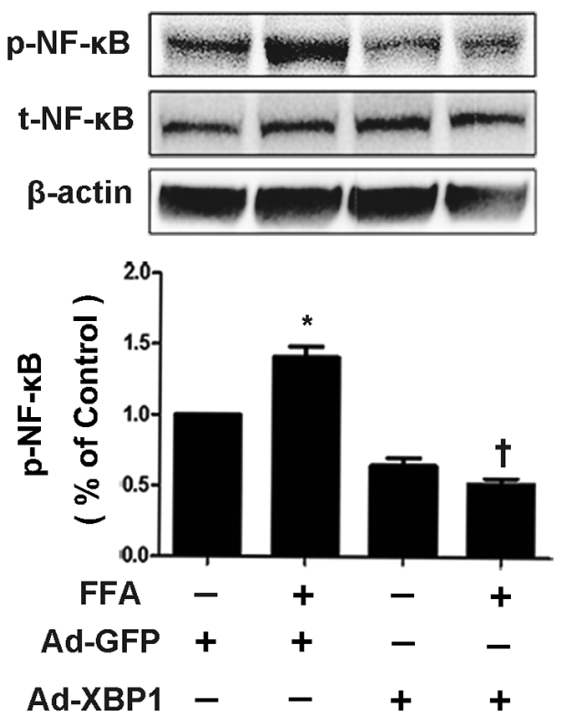

expression of XBP1 was achieved by infection of 3T3-L1 adipocytes with adenovirus encoding with XBP1 at a multiplicity of infection of 20 for $48 \mathrm{~h}$. Adenovirus encoding GFP (Ad-GFP) was used as control. After infection with Ad-GFP and Ad-XBP1, 3T3-L1 adipocytes were treated with FFA for $4 \mathrm{~h}$. Representative results were from three independent experiments. $* P<0.05 ; * * P<0.01$ versus Ad-GFP; ${ }^{\dagger} P<0.05 ;{ }^{\ddagger} P<0.01$ versus Ad-GFP+FFA 
FFA-induced phosphorylation of IKK by overexpression of XBP1 via adenovirus infection. As shown in Fig. 6, treatment with FFAs induced a time-dependent increase in the phosphorylation of IкB- $\alpha$, NF- $\kappa \mathrm{B}$ p 65 , and IKK $\alpha / \beta$ which is markedly reversed by Ad-XBP1 transfection in 3T3-L1 adipocytes as determined by western blot analysis (Fig. 6a-c), while the total protein expression levels of IKK- $\alpha / \beta$, IкB- $\alpha$, and NF- $\mathrm{KB}$ p65 remained unchanged. These results confirmed the reduction of the inflammatory effect and activation of the NF- $\kappa B$ pathway by FFAs in 3T3-L1 adipocytes, which may in part contribute to XBP1. Activation of XBP1 requires splicing by IRE1 $\alpha$. To further assess the mechanisms of how XBP1 regulates the IKK pathway, we examined the phosphorylation of IRE1 $\alpha$ in FFA-stimulated cells. Results of western blot analysis showed that transfection with XBP1siRNA led to upregulated phosphorylation of IRE1 in FFA-treated 3T3-L1 adipocytes, but the total level of IRE1 $\alpha$ had not been changed (Fig. 7).

In conclusion, our data indicate that ER stress preconditioning can protect 3T3-L1 adipocytes against FFAinduced inflammatory response by the induction of XBP1. The upregulation of XBP1 led to the inhibition of IRE1/
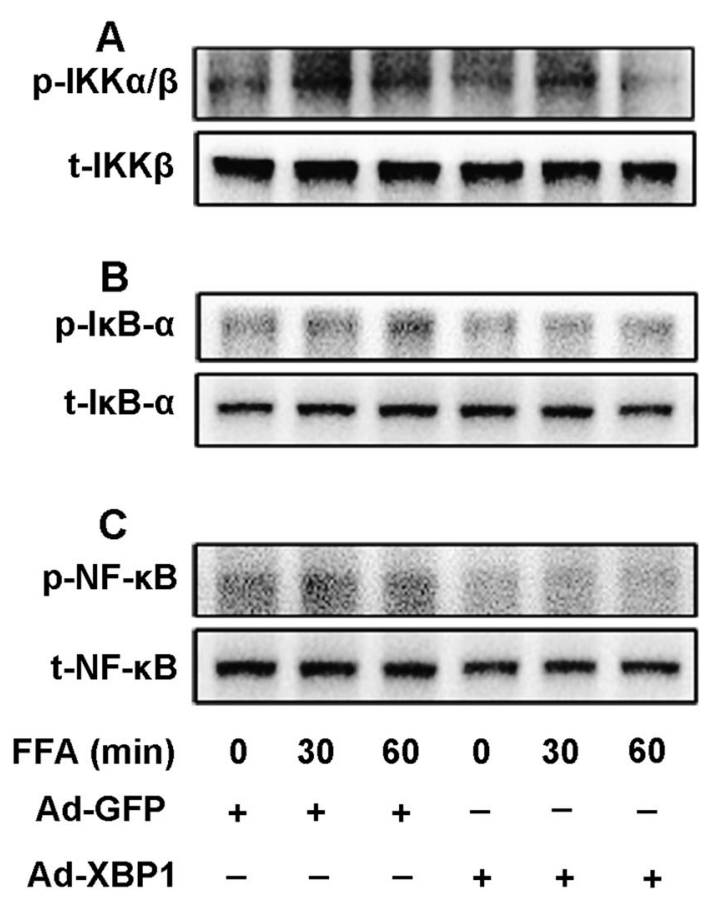

Fig. 6 Overexpression of XBP1 inhibited FFA-induced IKK activation. a-c Phosphorylation of IKK $\alpha / \beta$ at Ser176/180 (a), IאB- $\alpha$ at Ser32 (b), and NF- $\mathrm{KB}$ at Ser536 (c) were detected by Western blot analysis. 3T3-L1 adipocytes were infected with adenovirus encoding with XBP1 (Ad-XBP1) at a multiplicity of infection of 20 for $48 \mathrm{~h}$. Adenovirus encoding GFP (Ad-GFP) was used as control. After infection with Ad-GFP and Ad-XBP1, 3T3-L1 adipocytes were treated with $0.5 \mathrm{mM}$ FFA for up to $60 \mathrm{~min}$. Total IKK $\alpha$, IKK $\beta$, IкB- $\alpha$, and NF- $\kappa B$ were also determined. Representative results were from three independent experiments
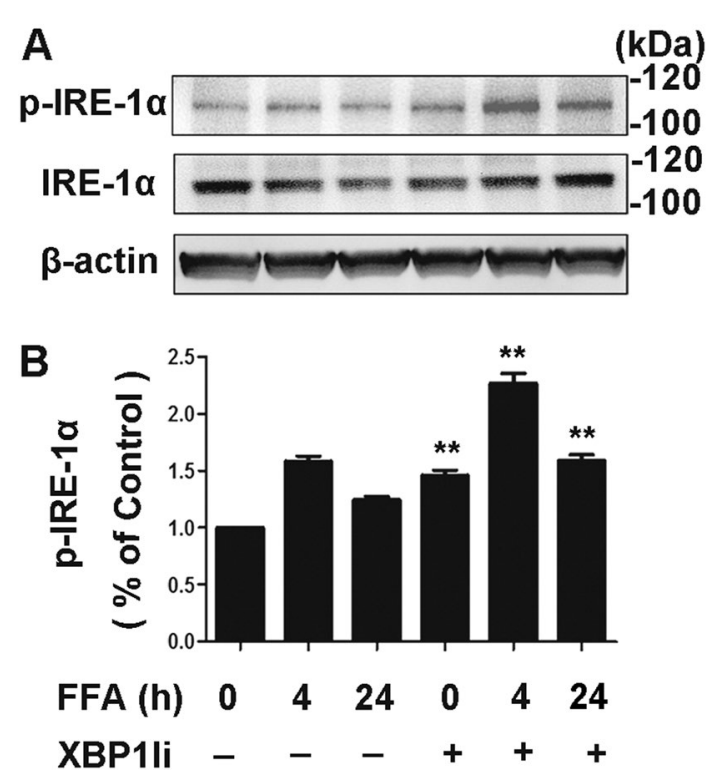

Fig. 7 XBP1 blocked FFA-induced IKK activation through inhibition of IRE phosphorylation. a, $\mathbf{b}$ Expressions of p-IRE-1 $\alpha$ (a), total phospho-IRE-1 $\alpha(\mathbf{b})$, and $\beta$-actin were determined by Western blot analysis and semi-quantified by densitometry. 3T3-L1 adipocytes were infected with XBP-1siRNA or control siRNA for $48 \mathrm{~h}$, followed by treatment with $0.5 \mathrm{mM}$ FFA for $4 \mathrm{~h}$ and $24 \mathrm{~h}$. Representative results were from three independent experiments. $* * P<0.01$ versus control or control siRNA

IKK/NF- $\kappa B$ pathway which in turn upregulates inflammatory gene expression, leading to adipocyte inflammation and obesity (Fig. 8).

\section{Discussion}

Obesity is strongly associated with cardiovascular disorders and diabetes. Recent studies have shown that excess energy intake and endogenous lipolysis of saturated fatty acids might increase the production of proinflammatory cytokines, including IL-6 and MCP-1 as well as activation of NF- $\mathrm{KB}$ signaling pathway, which partly contributed to adipocyte inflammation and obesity [34-36]. Inhibition of the NF- $\mathrm{KB}$ signaling pathway in 3T3-L1 adipocytes not only prevents adipocyte inflammation, but also ameliorates adipocyte dysfunction and increases insulin sensitivity [37, 38]. The NF- $\mathrm{KB}$ pathway is vital for the inflammatory response, which has been proven in several types of mature cells and various cellular signals. P-IKK $\alpha / \beta, p-I \kappa B \alpha$, and $p-p 65$ are the phosphorylated forms of three important proteins in the NF- $\kappa B$ pathway $[35,39,40]$. Our previous study demonstrated that $0.5 \mathrm{mM}$ FFAs upregulate phosphorylation of the NF- $\mathrm{kB}$ p65 subunit at Ser536, leading to overproduction of IL-6 and MCP-1 in 3T3-L1 adipocytes. In the present study, we demonstrated that preconditioning with ER 
Fig. 8 Role of XBP1 in Tunicamycin-induced ER stress preconditioning in FFAstimulated inflammatory gene expression in 3T3-L1 adipocytes. ER stress preconditioning via tunicamycin elevates XBP1s level, which inhibited FFA-stimulated NF- $\kappa$ B pathway and thus suppressed NF- $\mathrm{KB}$ activated inflammatory genes in 3T3-L1 adipocytes

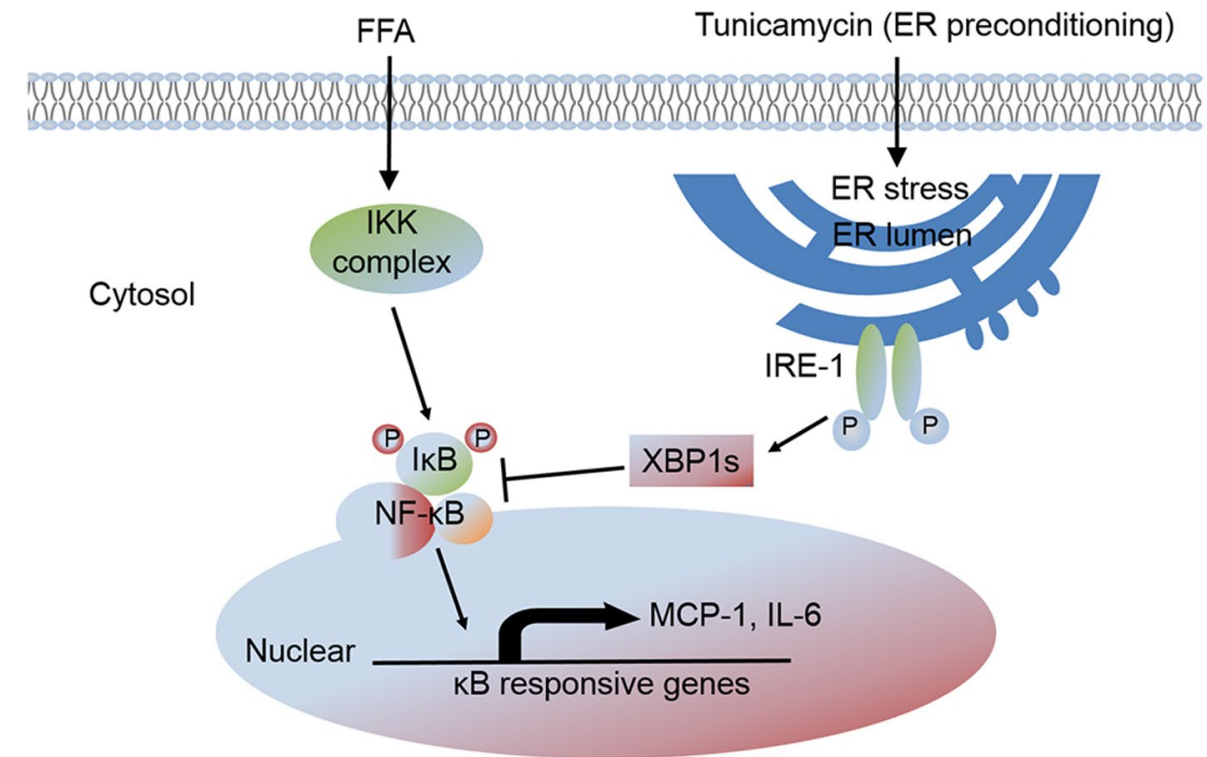

stress attenuated FFA-induced expression of inflammatory cytokines and NF- $\kappa \mathrm{B}$ activation in $3 \mathrm{~T} 3-\mathrm{L} 1$ adipocytes. In addition, the expressions of $\mathrm{p}-\mathrm{IKK} \alpha / \beta, \mathrm{p}-\mathrm{I} \kappa \mathrm{B} \alpha$, and $\mathrm{p}-\mathrm{p} 65$ in 3T3-L1 adipocytes were suppressed by ER stress preconditioning in time-dependent manners. Our results also indicate that activation of XBP1 is essential for the protective effects of ER stress preconditioning through mediation of the IRE1/IKK/NF- $\kappa \mathrm{B}$ pathway. This study for the first time elucidated a beneficial effect and involved mechanisms of ER stress preconditioning on regulation of adipocyte inflammation, which can help to elucidate the specific molecular mechanism of FFA-induced adipocyte inflammation and will provide theoretical basis for the treatment of obesity and metabolic disorders.

There is a close link between ER stress and the inflammatory response. In our previous study, we found that induction of ER stress contributes to retinal inflammation in diabetic retinopathy [41]. Jiao et al. [34] demonstrated that ER stress is a key mediator of FFA-induced inflammation in adipocytes together with PKR-like eukaryotic initiation factor $2 \alpha$ kinase, one of the three major ER stress sensor proteins. Accumulating evidence indicates a protective effect of ER stress preconditioning against the cytokine-induced inflammatory response. Yu et al. [42] demonstrated that preconditioning by ER stress suppresses acrylonitrile-induced cytotoxicity in primary rat astrocytes. Zhang et al. [25] showed that preconditioning with ER stress ameliorates inflammation in retinal endothelial cells by suppressing $\mathrm{NF}-\kappa \mathrm{B}-$ mediated adhesion molecule expression. Pretreatment with sub-nephritogenic doses of ER stress inducers tunicamycin or thapsigargin in rats mitigates mesangioproliferative glomerulonephritis [21]. These studies suggest a protective role of signaling pathways activated by ER stress against inflammatory conditions. In the present study, we showed that FFA-induced inflammatory cytokine production was significantly suppressed in ER stress-pretreated 3T3-L1 adipocytes, suggesting a protective role of ER stress preconditioning, which is in line with previous investigations.

XBP1s is an important gene that turns on transcription of a subset of ER chaperones genes in response to stress. Previous studies have reported a pivotal role of XBP1 in the development of several diseases such as Alzheimer's Disease [43], diabetic retinopathy [44], neurodegeneration [45], insulin resistance [46], and obesity [47]. Sha et al. [48] demonstrated that XBP1s improves glucose tolerance and insulin sensitivity in both lean and obese (ob/ob) mice by promoting adiponectin multimerization. Iwakoshi et al. [49] reported that XBP1 is required for plasma cell differentiation and the UPR. However, there are still some conflicting studies concerning the role of XBP1 during cell development. Claudio et al. [50] reported that XBP-1-deficient mice are more resistant to developing disease, which correlated with increased levels of autophagy in motoneurons. Our data indicated that overexpression of XBP1 suppressed FFA-induced secretion of inflammatory cytokines. Importantly, the present study demonstrated that pretreatment of 3T3-L1 adipocytes with the ER stress inducer tunicamycin upregulated XBP1, but not proinflammatory cytokines.

Previous groups have been reported that co-culture of 3T3-L1 adipocyte and macrophages activates the proinflammatory response and in turn upregulates the motility of macrophages [51]. In our study, we demonstrated that co-culture of 3T3-L1 adipocyte and macrophages increases the macrophages migration which is in line with the result of previous studies. Furthermore, we demonstrated that XBP1 siRNA blocks the inhibitory effect of ER stress preconditioning on FFA-induced macrophages migration which in turn augments the adipocyte inflammation. Little literatures have 
reported the effect of XBP1s on macrophage migration and adipocyte inflammation. Zhang and associates demonstrated that quinotrierixin, a specific inhibitor of XBP1 splicing, could increase the leukocyte adhesion to endothelial cells in the dose-dependent manner [25]. In addition, Ozcan et al. [52] showed that increased XBP1s activity leads to reduced ER stress, and improved insulin sensitivity and glucose homeostasis. Moreover, studies show that XBP1 deletion triggers feedback activation of its upstream enzyme, IRE1 $\alpha$ $[53,54]$. Sha et al. [55] reported that IRE1 $\alpha$ could promote glucose tolerance and insulin sensitivity through activated XBP1s in both starved and obese mice. In our study, we found that XBP1 negatively regulated IRE1 activation, a critical step for FFA-induced IKK and NF- $\mathrm{KB}$ activation, in both untreated and FFA-treated cells, which is consistent with a study by Zhang et al. [26]. Our findings are consistent with these results and highlight possible protective roles of anti-inflammatory and anti-obesity effects of XBP1s. However, the mechanisms by which XBP1 activates IRE1 $\alpha$ need to be investigated further.

In conclusion, our study demonstrates that preconditioning with ER stress attenuates FFA-induced inflammation in 3T3-L1 adipocytes. XBP1 plays an essential role in the protective effects of ER stress preconditioning and is required to inhibit FFA-induced inflammation mediated by the IRE1/IKK/NF- $\kappa B$ pathway. To our knowledge and as summarized in Fig. 8, this is the first study providing results of the protective role of ER stress preconditioning in FFA-induced adipocyte inflammation to exert the antiobesity effect. Identifying small molecules that enhance XBP1-mediated protective UPR signaling may provide a novel therapeutic strategy against adipocyte inflammation and obesity. Our study lacks in vivo data, which is a limitation. Thus, researches into the use of ob/ob mice or C57BL6/J mice with high-fat-diet-induced obesity as a target for metabolic diseases hold much promise.

Acknowledgements This work was supported by grants from the National Natural Science Foundation of China (Nos. 81300707; 81370447; 81770826 and 81830082).

\section{Compliance with ethical standards}

Conflict of interest The authors have no conflict of interest to declare.

Open Access This article is distributed under the terms of the Creative Commons Attribution 4.0 International License (http://creativeco mmons.org/licenses/by/4.0/), which permits unrestricted use, distribution, and reproduction in any medium, provided you give appropriate credit to the original author(s) and the source, provide a link to the Creative Commons license, and indicate if changes were made.

\section{References}

1. Xu H, Barnes GT, Yang Q, Tan G, Yang D, Chou CJ, Sole J, Nichols A, Ross JS, Tartaglia LA, Chen H (2003) Chronic inflammation in fat plays a crucial role in the development of obesity-related insulin resistance. J Clin Invest 112:1821-1830. https://doi.org/10.1172/jci19451

2. Wellen KE, Hotamisligil GS (2003) Obesity-induced inflammatory changes in adipose tissue. J Clin Invest 112:1785-1788. https://doi.org/10.1172/jci20514

3. Fischer IP, Irmler M, Meyer CW, Sachs SJ, Neff F, Hrabe de Angelis M, Beckers J, Tschop MH, Hofmann SM, Ussar S (2018) A history of obesity leaves an inflammatory fingerprint in liver and adipose tissue. Int J Obes (Lond) 42:507-517. https ://doi.org/10.1038/ijo.2017.224

4. McCall KD, Holliday D, Dickerson E, Wallace B, Schwartz AL, Schwartz C, Lewis CJ, Kohn LD, Schwartz FL (2010) Phenylmethimazole blocks palmitate-mediated induction of inflammatory cytokine pathways in 3T3L1 adipocytes and RAW 264.7 macrophages. J Endocrinol 207:343-353. https:// doi.org/10.1677/joe-09-0370

5. Vitseva OI, Tanriverdi K, Tchkonia TT, Kirkland JL, McDonnell ME, Apovian CM, Freedman J, Gokce N (2008) Inducible Tolllike receptor and NF-kappaB regulatory pathway expression in human adipose tissue. Obesity (Silver Spring) 16:932-937. https://doi.org/10.1038/oby.2008.25

6. Wang M, Chen Y, Xiong Z, Yu S, Zhou B, Ling Y, Zheng Z, Shi G, Wu Y, Qian X (2017) Ginsenoside Rb1 inhibits free fatty acids induced oxidative stress and inflammation in 3T3L1 adipocytes. Mol Med Rep 16:9165-9172. https://doi.org/10.3892/ mmr.2017.7710

7. Odegaard JI, Ricardo-Gonzalez RR, Goforth MH, Morel CR, Subramanian V, Mukundan L, Red Eagle A, Vats D, Brombacher F, Ferrante AW, Chawla A (2007) Macrophage-specific PPARgamma controls alternative activation and improves insulin resistance. Nature 447:1116-1120. https://doi.org/10.1038/ nature 05894

8. Kirk EA, Sagawa ZK, McDonald TO, O'Brien KD, Heinecke JW (2008) Monocyte chemoattractant protein deficiency fails to restrain macrophage infiltration into adipose tissue [corrected]. Diabetes 57:1254-1261. https://doi.org/10.2337/db07-1061

9. Akbari M, Hassan-Zadeh V (2018) IL-6 signalling pathways and the development of type 2 diabetes. Inflammopharmacology 26:685-698. https://doi.org/10.1007/s10787-018-0458-0

10. Boden G (2011) Obesity, insulin resistance and free fatty acids. Curr Opin Endocrinol Diabetes Obes 18:139-143. https://doi. org/10.1097/MED.0b013e3283444b09

11. Ron D, Walter P (2007) Signal integration in the endoplasmic reticulum unfolded protein response. Nat Rev Mol Cell Biol 8:519-529. https://doi.org/10.1038/nrm2199

12. Gregor MF, Hotamisligil GS (2007) Thematic review series: adipocyte biology. Adipocyte stress: the endoplasmic reticulum and metabolic disease. J Lipid Res 48:1905-1914. https://doi. org/10.1194/jlr.R700007-JLR200

13. Hotamisligil GS (2008) Inflammation and endoplasmic reticulum stress in obesity and diabetes. Int J Obes (Lond) 32(Suppl 7):S52-S54. https://doi.org/10.1038/ijo.2008.238

14. Hotamisligil GS (2010) Endoplasmic reticulum stress and the inflammatory basis of metabolic disease. Cell 140:900-917. https://doi.org/10.1016/j.cell.2010.02.034

15. Cox JS, Chapman RE, Walter P (1997) The unfolded protein response coordinates the production of endoplasmic reticulum protein and endoplasmic reticulum membrane. Mol Biol Cell 8:1805-1814 
16. Paschen W (2004) Endoplasmic reticulum dysfunction in brain pathology: critical role of protein synthesis. Curr Neurovasc Res $1: 173-181$

17. Feldman DE, Chauhan V, Koong AC (2005) The unfolded protein response: a novel component of the hypoxic stress response in tumors. Mol Cancer Res 3:597-605. https://doi. org/10.1158/1541-7786.MCR-05-0221

18. Xu C, Bailly-Maitre B, Reed JC (2005) Endoplasmic reticulum stress: cell life and death decisions. J Clin Invest 115:26562664. https://doi.org/10.1172/JCI26373

19. Eizirik DL, Cardozo AK, Cnop M (2008) The role for endoplasmic reticulum stress in diabetes mellitus. Endocr Rev 29:42-61. https://doi.org/10.1210/er.2007-0015

20. Iwakoshi NN, Lee AH, Vallabhajosyula P, Otipoby KL, Rajewsky K, Glimcher LH (2003) Plasma cell differentiation and the unfolded protein response intersect at the transcription factor XBP-1. Nat Immunol 4:321-329. https://doi.org/10.1038/ni907

21. Inagi R, Kumagai T, Nishi H, Kawakami T, Miyata T, Fujita T, Nangaku M (2008) Preconditioning with endoplasmic reticulum stress ameliorates mesangioproliferative glomerulonephritis. J Am Soc Nephrol 19:915-922. https://doi.org/10.1681/ ASN.2007070745

22. Hung CC, Ichimura T, Stevens JL, Bonventre JV (2003) Protection of renal epithelial cells against oxidative injury by endoplasmic reticulum stress preconditioning is mediated by ERK1/2 activation. J Biol Chem 278:29317-29326. https://doi. org/10.1074/jbc.M302368200

23. Peyrou M, Cribb AE (2007) Effect of endoplasmic reticulum stress preconditioning on cytotoxicity of clinically relevant nephrotoxins in renal cell lines. Toxicol In Vitro 21:878-886. https://doi.org/10.1016/j.tiv.2007.03.001

24. Leonard A, Paton AW, El-Quadi M, Paton JC, Fazal F (2014) Preconditioning with endoplasmic reticulum stress ameliorates endothelial cell inflammation. PLoS ONE 9:e110949. https:// doi.org/10.1371/journal.pone.0110949

25. Li J, Wang JJ, Zhang SX (2011) Preconditioning with endoplasmic reticulum stress mitigates retinal endothelial inflammation via activation of $\mathrm{X}$-box binding protein 1. J Biol Chem 286:4912-4921. https://doi.org/10.1074/jbc.M110.199729

26. Wang M, Wang JJ, Li J, Park K, Qian X, Ma JX, Zhang SX (2009) Pigment epithelium-derived factor suppresses adipogenesis via inhibition of the MAPK/ERK pathway in 3T3-L1 preadipocytes. Am J Physiol Endocrinol Metab 297:E1378-E1387. https://doi.org/10.1152/ajpendo.00252.2009

27. Suganami T, Nishida J, Ogawa Y (2005) A paracrine loop between adipocytes and macrophages aggravates inflammatory changes: role of free fatty acids and tumor necrosis factor alpha. Arterioscler Thromb Vasc Biol 25:2062-2068. https:// doi.org/10.1161/01.atv.0000183883.72263.13

28. Wang Y, Chen Y, Zhou Q, Xu J, Qian Q, Ni P, Qian Y (2018) Mild endoplasmic reticulum stress protects against lipopolysaccharide-induced astrocytic activation and blood-brain barrier hyperpermeability. Front Cell Neurosci 12:222. https://doi. org/10.3389/fncel.2018.00222

29. Liu D, Liu X, Zhou T, Yao W, Zhao J, Zheng Z, Jiang W, Wang F, Aikhionbare FO, Hill DL, Emmett N, Guo Z, Wang D, Yao X, Chen Y (2016) IRE1-RACK1 axis orchestrates ER stress preconditioning-elicited cytoprotection from ischemia/reperfusion injury in liver. J Mol Cell Biol 8:144-156. https://doi. org/10.1093/jmcb/mjv066

30. Zarrabi K, Dufour A, Li J, Kuscu C, Pulkoski-Gross A, Zhi J, Hu Y, Sampson NS, Zucker S, Cao J (2011) Inhibition of matrix metalloproteinase 14 (MMP-14)-mediated cancer cell migration. J Biol Chem 286:33167-33177. https://doi.org/10.1074/ jbc.M111.256644
31. Svedberg J, Bjorntorp P, Smith U, Lonnroth P (1990) Free-fatty acid inhibition of insulin binding, degradation, and action in isolated rat hepatocytes. Diabetes 39:570-574

32. Piperi C, Adamopoulos C, Papavassiliou AG (2016) XBP1: a pivotal transcriptional regulator of glucose and lipid metabolism. Trends Endocrinol Metab 27:119-122. https://doi. org/10.1016/j.tem.2016.01.001

33. Sha H, Yang L, Liu M, Xia S, Liu Y, Liu F, Kersten S, Qi L (2014) Adipocyte spliced form of X-box-binding protein 1 promotes adiponectin multimerization and systemic glucose homeostasis. Diabetes 63:867-879. https://doi.org/10.2337/db13-1067

34. Jiao P, Ma J, Feng B, Zhang H, Diehl JA, Chin YE, Yan W, Xu H (2011) FFA-induced adipocyte inflammation and insulin resistance: involvement of ER stress and IKKbeta pathways. Obesity (Silver Spring) 19:483-491. https://doi.org/10.1038/oby.2010.200

35. Liu T, Zhang L, Joo D, Sun SC (2017) NF-kappaB signaling in inflammation. Signal Transduct Target Ther 2. https://doi. org/10.1038/sigtrans.2017.23

36. Sanyal A, Naumann J, Hoffmann LS, Chabowska-Kita A, Ehrlund A, Schlitzer A, Arner P, Bluher M, Pfeifer A (2017) Interplay between obesity-induced inflammation and cGMP signaling in white adipose tissue. Cell Rep 18:225-236. https://doi. org/10.1016/j.celrep.2016.12.028

37. Krinninger P, Brunner C, Ruiz PA, Schneider E, Marx N, ForystLudwig A, Kintscher U, Haller D, Laumen H, Hauner H (2011) Role of the adipocyte-specific NF-kappaB activity in the regulation of IP-10 and T cell migration. Am J Physiol Endocrinol Metab 300:E304-E311. https://doi.org/10.1152/ajpendo.00143 .2010

38. Usuki F, Fujimura M, Yamashita A (2013) Endoplasmic reticulum stress preconditioning attenuates methylmercury-induced cellular damage by inducing favorable stress responses. Sci Rep 3:2346. https://doi.org/10.1038/srep02346

39. Israel A (2010) The IKK complex, a central regulator of NFkappaB activation. Cold Spring Harb Perspect Biol 2:a000158. https://doi.org/10.1101/cshperspect.a000158

40. Baeuerle PA (1998) IkappaB-NF-kappaB structures: at the interface of inflammation control. Cell 95:729-731

41. Li J, Wang JJ, Yu Q, Wang M, Zhang SX (2009) Endoplasmic reticulum stress is implicated in retinal inflammation and diabetic retinopathy. FEBS Lett 583:1521-1587. https://doi.org/10.1016/j. febslet.2009.04.007

42. Yu B, Wenjun Z, Changsheng Y, Yuntao F, Jing M, Ben L, Hai Q, Guangwei X, Suhua W, Fang L, Aschner M, Rongzhu L (2016) Preconditioning of endoplasmic reticulum stress protects against acrylonitrile-induced cytotoxicity in primary rat astrocytes: the role of autophagy. Neurotoxicology 55:112-121. https://doi. org/10.1016/j.neuro.2016.05.020

43. Martinez G, Vidal RL, Mardones P, Serrano FG, Ardiles AO, Wirth C, Valdes P, Thielen P, Schneider BL, Kerr B, Valdes JL, Palacios AG, Inestrosa NC, Glimcher LH, Hetz C (2016) Regulation of memory formation by the transcription factor XBP1. Cell Rep 14:1382-1394. https://doi.org/10.1016/j.celrep.2016.01.028

44. McLaughlin T, Falkowski M, Park JW, Keegan S, Elliott M, Wang JJ, Zhang SX (2018) Loss of XBP1 accelerates age-related decline in retinal function and neurodegeneration. Mol Neurodegener 13:16. https://doi.org/10.1186/s13024-018-0250-z

45. Bobkova NV, Evgen'ev M, Garbuz DG, Kulikov AM, Morozov A, Samokhin A, Velmeshev D, Medvinskaya N, Nesterova I, Pollock A, Nudler E (2015) Exogenous Hsp70 delays senescence and improves cognitive function in aging mice. Proc Natl Acad Sci USA 112:16006-16011. https://doi.org/10.1073/pnas.15161 31112

46. Liu DH, Chen YM, Liu Y, Hao BS, Zhou B, Wu L, Wang M, Chen L, Wu WK, Qian XX (2011) Rb1 protects endothelial cells from 
hydrogen peroxide-induced cell senescence by modulating redox status. Biol Pharm Bull 34:1072-1077

47. Yang J, Wu X, Wu X, Zhou D, Lin T, Ding S, Zhang Y, Xue J, Zhuge Q (2017) The multiple roles of XBP1 in regulation of glucose and lipid metabolism. Curr Protein Pept Sci 18:630-635. https://doi.org/10.2174/1389203717666160627085011

48. Sha H, He Y, Chen H, Wang C, Zenno A, Shi H, Yang X, Zhang X, Qi L (2009) The IRE1alpha-XBP1 pathway of the unfolded protein response is required for adipogenesis. Cell Metab 9:556564. https://doi.org/10.1016/j.cmet.2009.04.009

49. Iwakoshi NN, Lee AH, Glimcher LH (2003) The X-box binding protein- 1 transcription factor is required for plasma cell differentiation and the unfolded protein response. Immunol Rev 194:29-38

50. Hetz C, Thielen P, Matus S, Nassif M, Court F, Kiffin R, Martinez G, Cuervo AM, Brown RH, Glimcher LH (2009) XBP-1 deficiency in the nervous system protects against amyotrophic lateral sclerosis by increasing autophagy. Genes Dev 23:2294-2306. https://doi.org/10.1101/gad.1830709

51. Ceppo F, Berthou F, Jager J, Dumas K, Cormont M, Tanti JF (2014) Implication of the Tpl2 kinase in inflammatory changes and insulin resistance induced by the interaction between adipocytes and macrophages. Endocrinology 155:951-964. https://doi. org/10.1210/en.2013-1815

52. Liu J, Ibi D, Taniguchi K, Lee J, Herrema H, Akosman B, Mucka P, Salazar Hernandez MA, Uyar MF, Park SW, Karin M, Ozcan
U (2016) Inflammation improves glucose homeostasis through IKKbeta-XBP1s interaction. Cell 167:1052-1066.e18. https://doi. org/10.1016/j.cell.2016.10.015

53. Calfon M, Zeng H, Urano F, Till JH, Hubbard SR, Harding HP, Clark SG, Ron D (2002) IRE1 couples endoplasmic reticulum load to secretory capacity by processing the XBP-1 mRNA. Nature 415:92-96. https://doi.org/10.1038/415092a

54. Yoshida H, Matsui T, Yamamoto A, Okada T, Mori K (2001) XBP1 mRNA is induced by ATF6 and spliced by IRE1 in response to ER stress to produce a highly active transcription factor. Cell 107:881-891

55. Shao M, Shan B, Liu Y, Deng Y, Yan C, Wu Y, Mao T, Qiu Y, Zhou Y, Jiang S, Jia W, Li J, Li J, Rui L, Yang L, Liu Y (2014) Hepatic IRE1alpha regulates fasting-induced metabolic adaptive programs through the XBP1s-PPARalpha axis signalling. Nat Commun 5:3528. https://doi.org/10.1038/ncomms4528

Publisher's Note Springer Nature remains neutral with regard to jurisdictional claims in published maps and institutional affiliations. 